Revue internationale P.M.E.

Économie et gestion de la petite et moyenne entreprise

\title{
De la cotraitance au management relationnel: le cas d'une coopérative artisanale du secteur du bâtiment
}

\section{Katia Richomme}

Volume 14, numéro 2, 2001

URI : https://id.erudit.org/iderudit/1008694ar

DOI : https://doi.org/10.7202/1008694ar

Aller au sommaire du numéro

Éditeur(s)

Presses de l’Université du Québec

ISSN

0776-5436 (imprimé)

1918-9699 (numérique)

Découvrir la revue

Citer cette note

Richomme, K. (2001). De la cotraitance au management relationnel: le cas d'une coopérative artisanale du secteur du bâtiment. Revue internationale P.M.E., 14(2), 109-131. https://doi.org/10.7202/1008694ar
Résumé de l'article

Les recherches dans le domaine des coopérations interfirmes se sont essentiellement intéressées aux grandes entreprises, parfois aux PME, mais beaucoup plus rarement aux très petites entreprises artisanales. Nous visons à apporter une contribution au management relationnel à travers une étude qualitative portant sur une coopération formalisée et réussie. L'intérêt de cette coopérative artisanale est de démontrer qu'une stratégie de cotraitance imposée par le marché peut conduire à une véritable alliance, basée sur la gestion de leurs relations spécifiques, coordonnée par la confiance et l'acceptation d'une constante évolution. 


\title{
De la cotraitance au management relationnel: le cas d'une coopérative artisanale du secteur du bâtiment
}

\author{
Katia RICHOMME \\ École nationale supérieure des industries chimiques
}

\section{MOTS CLÉS}

\section{Coopérative artisanale - Cotraitance - Solidarité \\ Relationnel - Confiance}

\begin{abstract}
RÉSUMÉ
Les recherches dans le domaine des coopérations interfirmes se sont essentiellement intéressées aux grandes entreprises, parfois aux PME, mais beaucoup plus rarement aux très petites entreprises artisanales. Nous visons à apporter une contribution au management relationnel à travers une étude qualitative portant sur une coopération formalisée et réussie. L'intérêt de cette coopérative artisanale est de démontrer qu'une stratégie de cotraitance imposée par le marché peut conduire à une véritable alliance, basée sur la gestion de leurs relations spécifiques, coordonnée par la confiance et l'acceptation d'une constante évolution.
\end{abstract}

\section{L'AUTEURE}

KaTIA RICHOMme est maître de conférences à l'École nationale supérieure des industries chimiques de Nancy (ENSIC), laquelle dépend de l'Institut national polytechnique de Lorraine (INPL), titulaire d'un doctorat en sciences de gestion et membre de l'ERFI (Équipe de recherche sur la firme et l'industrie) à l'Université de Montpellier I.

Ses recherches portent principalement sur les aspects stratégiques dans les petites entreprises et sur les liens existant entre les dirigeants et leur mode de gestion de l'entreprise. Adresse : Université de Montpellier I, avenue de la Mer, Espace Richter, Bâtiment E, B.P. 9659, 34054 Montpellier Cedex 1, France. Courriel : <katia.richomme@free.fr>. 


\begin{abstract}
Research in inter-firms cooperations mainly focused on large firms, sometimes on SMEs, but sparsely on very small craft industry. We tried to set up a contribution to the professional relationship management, through a qualitative study of a formalized and successful co-operation. The point in this craftsmanship cooperative is to demonstrate that a co-contracting strategy imposed by the market can lead to a genuine partnership, based on specific professional relationship management, along with their inner trust and on the acceptance of a perpetual evolution.

\section{RESUMEN}

Las investigaciones en el campo de las cooperaciones interfirmas se han interesado en las grandes empresas, de vez en cuando a las PyMEs, pero mucho rara vez a las pequeñísimas empresas del artesano. Proponemos una contribution a la gestión de las relaciones, a través de un estudio cualitativo de una cooperación formalizada y conseguida. El interés de este cooperativa artesanal es demostrar que una estrategia de co-contratado impuesto por el mercado puede conducir a una legítima alianza, basado en la gestión de los suyos relaciones específicas, coordinado por la confianza y por la aceptación de una constante evolución.
\end{abstract}

\title{
ZUSAMMENFASSUNG
}

Die Untersuchungen im Bereich der betrieblichen Kooperation haben sich in erster Linie den grossen Unternehmen, manchmal auch den KMU gewidmet, aber viel weniger den kleinen gewerblichen Unternehmen. In unserem Interesse ist es, einen Beitrag zu leisten zum Beziehungsmanagement mit einer qualitativen Untersuchung, die auf einer erfolgreichen und formalisierten Zusammenarbeit besteht. Das interessante an dieser gewerblichen Zusammenarbeit ist die Tatsache, dass eine Strategie diktiert durch den Markt zu einer effektiven Allianz führen kann. Diese Allianz basiert aber auf einer spezifischen Führung, welche koordiniert und durch das Vertrauen und die Akzeptanz unterstützt wird.

\section{Introduction}

Les théories les plus récentes sur la stratégie (Doz et Hamel, 1998 ; Koenig, 1996) mettent l'accent sur les stratégies relationnelles, soit celles qui se développent précisément dans un contexte d'entente plutôt que d'affrontement. En 1985, Joffre et Koenig avaient souligné le décentrage de la pensée stratégique et, plus précisément, les nouvelles orientations de cette pensée par l'opposition compétition / coopération. Les stratégies d'alliance ou de coopération en sont les illustrations les plus connues aujourd'hui. «Une stratégie est dite relationnelle lorsqu'elle se fonde non pas sur la loi de la concurrence, mais sur des relations privilégiées que l'entreprise établit avec certains partenaires de son environnement. La notion de concurrence n'en est pas totalement absente, mais elle est forcément secondaire par rapport à un accord scellé de gré à gré et échappant aux règles normales du marché » (Stratégor, 1991).

Revue internationale P.M.E., vol. 14, nº 2, 2001 
Les stratégies relationnelles accordent une place déterminante à certains rapports privilégiés, dépassant les rapports marchands, avec certains partenaires. Elles permettent de se procurer des ressources et des compétences par des marchés captifs, des accords préférentiels et des solidarités diverses (Bréchet, 1996).

Nombre d'auteurs se sont intéressés aux alliances stratégiques, mais peu se sont penchés sur l'association de ce concept à la très petite entreprise et encore moins à l'entreprise artisanale. Pourtant, les groupements d'artisans, même s'ils sont « peu nombreux, ambigus et à demi efficaces » (Louart, 1980) constituent un enjeu puissant. Dans toute démarche de regroupement d'artisans, l'intérêt réside dans la réunion de moyens techniques et humains collectifs afin de créer une nouvelle dynamique économique ; ce sont des réponses face au risque de pertes de marchés. L'analyse du cas «Artisans Services Bâtiment» nous a conduite à repositionner certaines variables issues de la littérature et à en insérer de nouvelles, dans le cadre des entreprises de très petites tailles ne souhaitant ni croître ni fusionner, mais obtenir dans la coopération de nouvelles compétences.

Nous proposons de nous inspirer de l'approche relationnelle, qui présente une critique idéologique des modèles concurrentiels. Et, plus précisément, en appliquant le modèle issu à la fois des travaux de Perlmutter et Heenan (1986), qui ont étudié les conditions nécessaires aux succès des partenariats stratégiques globaux et du Business Intelligence Program conçu par le Standford Research Institute, analysant le succès d'une alliance.

Après avoir présenté les principales conditions relevées par ces chercheurs et tenté d'en réduire les limites, créées d'ailleurs par l'application à des entreprises de grandes tailles, nous proposons la transposition de ce modèle à une coopérative artisanale, à travers une étude qualitative portant sur une coopérative régionale.

\section{Analyses descriptive et explicative du cas «Artisans Services Bâtiment » par une confrontation des théories sur les relations entre firmes}

Notre recherche repose sur le postulat initial que, dans les très petites entreprises où le propriétaire se confond presque systématiquement avec le dirigeant, le chef d'entreprise cherche de préférence à établir des relations privilégiées avec les autres acteurs, au lieu de se placer régulièrement en situation d'affrontement. Le courant de la théorie des besoins (McClelland, 1953 ; Maslow, 1954 ; Herzberg, 1966) démontre parfaitement que l'individu peut être motivé par des besoins de sécurité. Même si cette théorie présente un certain nombre de limites, d'autres auteurs ont insisté sur la prépondérance des relations (Alderfer, 1972) et de la sécurité (Porter, 1964). Ces relations peuvent prendre différentes formes selon le but recherché, les options stratégiques envisagées, le positionnement de l'entreprise sur un marché et le niveau de ses compétences ou de ses ressources.

Revue internationale P.M.E., vol. 14, nº 2, 2001

(C) 2001 - Presses de l'Université du Québec

Édifice Le Delta I, 2875, boul. Laurier, bureau 450, Sainte-Foy, Québec G1V 2M2 - Tél. : (418) 657-4399 - www.puq.uquebec.ca

Tiré de : Revue internationale P.M.E., vol. 14, $\mathrm{n}^{\circ}$ 2, sous la direction de Pierre-André Julien. 


\subsection{Le cadre conceptuel des relations entre firmes}

Les théories économiques classique et néoclassique de la concurrence, l'économie industrielle, l'analyse stratégique de l'industrie et de la concurrence ou les approches classiques du management stratégique tentent en général « d'analyser, de formaliser et de prescrire les comportements des entreprises en situation d'affrontement concurrentiel pur et non pas en situation de coopération» (Garrette, 1995). Cette limite essentielle, largement reconnue (Joffre et Koenig, 1985; Anastassopoulos et al., 1985; Bréchet, 1996) et issue de l'idéologie nord-américaine, a conduit à un élargissement de la pensée stratégique, amenant les chercheurs à s'intéresser aux phénomènes de coopération, s'éloignant alors de l'approche traditionnelle fondée sur la libre concurrence et les pures relations de marché. Cependant, le propos n'est pas d'ignorer les stratégies classiques d'affrontement, mais de mettre en évidence la possibilité d'une coexistence de ces deux formes de comportement. Autre limite importante, ces théories abordent l'entreprise comme un acteur en soi $^{1}$, sans rendre compte de son fonctionnement réel et en réduisant la complexité des relations humaines entre les individus de l'organisation et/ou interfirmes.

Malgré l'intérêt de la théorie de l'agence et, plus particulièrement, dans la mise en évidence des coûts d'agence inhérents à toute relation de coopération, au sens de Jensen et Meckling (1976), nous avons délibérément choisi de ne pas nous placer dans l'approche transactionnelle qui remet en cause les hypothèses de la théorie économique classique (Williamson, 1975 ; Ulrich, 1983), cherche à réduire les incertitudes (Arndt, 1979), les risques ou les coûts (Zeithaml et Zeithaml, 1984) et qui se focalisent essentiellement sur le marché et la hiérarchie (Koenig et Van Wijk, 1992 ; Ring et Van de Ven, 1992 ; Zaheer et Venkatram, 1995).

Nous réfutons aussi, par rapport à notre objet d'étude, les alliances comme forme nouvelle de la concurrence (Jacquemin et Remiche, 1988 ; Hamel, Doz et Prahalad, 1989) fondées sur le postulat suivant : les accords induisent une symétrie des relations telle qu'il y a forcément, comme dans l'approche traditionnelle de la stratégie, un gagnant et un perdant. Enfin, dernière objection posée a priori, la coopérative artisanale ne cherche pas dans la coopération une implication en termes d'innovations technologiques (Teece, 1986).

Partant, nous nous positionnons dans une approche relationnelle, essentiellement défendue par l'équipe du Strategor, où ce type de stratégie permet de maximiser la sécurité, c'est-à-dire «le degré de certitude qui caractérise la relation entre

1. Cette conception a été nuancée en 1976 par Jensen et Meckling dans l'article «Theory of the firm: Managerial behavior, agency costs and ownership structure », lorsqu'ils précisent que «la firme n'est pas un individu [...] mais une fiction légale», p. 311. 
l'entreprise et chacun de ses partenaires privilégiés ${ }^{2} »$. Cette théorie des relations rejoint la conception « humaniste » au sens d'Allaire et de Firsirotu (1993), tout en intégrant la relation humaine à l'identité de l'entreprise, aux processus de prise de décision, aux structures d'organisation et à l'analyse stratégique.

\subsection{Les pratiques de coopération dans la société coopérative "ASB »}

La littérature consacrée aux différentes stratégies de coopération s'attache essentiellement à démontrer aux dirigeants, managers, des grandes entreprises que cette stratégie est la réponse aux changements intensifs et rapides de l'activité économique, de la technologie et de la mondialisation. Ainsi, Doz et Hamel (1998) insistent sur l'obligation d'alliance dans cette course pour la conquête du futur où une entreprise isolée est condamnée à court terme. Cependant, le cadre de leurs travaux se situe dans une optique de compétition internationale et, plus spécifiquement, dans la création de valeur par le partenariat interentreprises de grande dimension, voire entre une grande entreprise et une plus petite.

L'objet de notre contribution, la coopérative artisanale, s'inscrit dans une tout autre approche, puisqu'il s'agit de l'association de très petites entreprises, encore peu étudiées comparativement aux PME (Puthod, 1995, 1998; Wacheux, 1993) et dans un secteur ayant suscité jusqu'ici assez peu de travaux en gestion (Simeoni, 1999 ; Louart, 1980). Cette section va permettre d'identifier les stratégies mises en place par les membres actuels de la structure coopérative afin non seulement de visualiser leur évolution, mais aussi et surtout de comprendre les processus de mise en œuvre des changements.

\subsubsection{De la cotraitance subie à la cotraitance délibérée, comme première étape de la coopération}

Selon Martinet (1983), les formes de coopérations interentreprises, appelées «stratégie d'impartition », proposent une alternative stratégique à la lutte entre concurrents. Et, dans les différents types d'impartition, la cotraitance voit les partenaires se partager la réalisation d'une production ou s'associer pour certaines opérations dans le cadre d'un groupement d'intérêt économique (G.I.E.). Effectivement, avant de créer la coopérative actuelle, les artisans étudiés avaient créé une première association, sous forme de groupement d'intérêt économique, afin de réaliser des maisons clés en main.

2. STRATÉGOR, Stratégie, structure, décision, identité : Politique générale d'entreprise, Paris, InterÉditions, 1991, p. 185.

Revue internationale P.M.E., vol. 14, nº 2, 2001

(C) 2001 - Presses de l'Université du Québec

Édifice Le Delta I, 2875, boul. Laurier, bureau 450, Sainte-Foy, Québec G1V 2M2 • Tél. : (418) 657-4399 - www.puq.uquebec.ca

Tiré de : Revue internationale P.M.E., vol. 14, $\mathrm{n}^{\circ} 2$, sous la direction de Pierre-André Julien. 
SCHÉMA 1

Représentation du contexte concurrentiel des entreprises artisanales avant la création du groupement

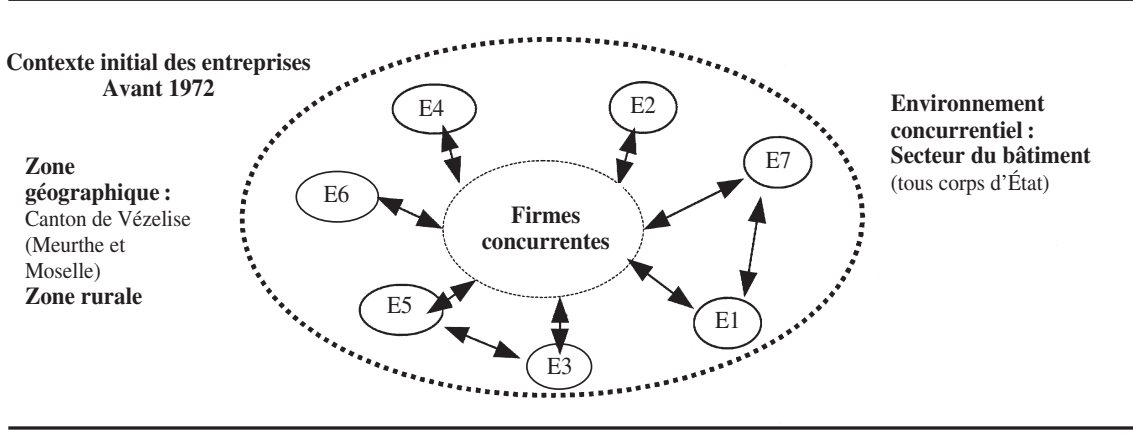

L'interdépendance des firmes paraît intimement liée à la géographie des marchés (rivaux proches) et à l'appartenance des offreurs au même groupe de concurrence. Chaque entreprise se trouve isolée dans une situation de compétition par rapport à des entreprises concurrentes (E1-E7 ou E3-E5) sur le même marché (plomberie et menuiserie), ou sur des marchés complémentaires qu'elle ne peut affronter par manque de moyens. La concurrence entre les firmes s'exerce selon l'interaction schématisée par les flèches. Les firmes repérées dans notre recherche, bien qu'évoluant dans le même secteur d'activité, à savoir le bâtiment, ne sont pas toutes en situation de concurrence, puisque certaines possèdent une mission et un métier différents des autres.

Selon Gervais (1995), la cotraitance, bien que considérée par Barreyre (1968, 1982) comme relevant de l'impartition, est en fait à la limite de l'impartition, de l'alliance et de la symbiose. Les partenaires se partagent la responsabilité de la réalisation, quitte à ce que l'un d'eux ressorte comme chef de file (l'entreprise principale). Effectivement, nous avons systématiquement constaté dans notre étude l'existence d'une entreprise «centrale» : au cours de l'existence du G.I.E., c'est l'architecte qui représentait le centre du système stratégique. "C'était parti de l'architecte parce les plans étaient faits par lui, tout était fait par lui; donc, c'est lui qui avait les demandes des clients, ou quelquefois le maçon qui emmenait le client chez l'architecte et ensuite c'était éclaté auprès des entreprises. » Puis, dans la coopérative, c'est l'entreprise d'agencement qui est «le point de chute pour les artisans et pour les clients », «un seul interlocuteur, toujours quelqu'un, toujours joignable, avec une réponse technique pour tous les corps de métiers».

Le principe de cotraitance, traditionnel dans le secteur du bâtiment, est basé soit sur l'adjudication d'un marché, soit sur l'attribution par un donneur d'ordres. Dans la pratique, la cotraitance peut prendre deux formes selon la stratégie de l'entreprise concernée : 
- une stratégie de cotraitance «subie», au sens où le maître d'ouvrage va demander l'alliance des entreprises retenues à l'appel d'offres, dans le but de les engager solidairement (Wacheux, 1993);

- une stratégie de cotraitance « délibérée », dans ce cas, la connaissance préalable des attributions de marchés peut conduire des entreprises à s'associer avant la proposition d'offres, pour pouvoir choisir leurs partenaires.

«Dans les années 1950-1960, [...] il n'y avait pas de communication entre les artisans, pas de suivi de chantier et pas de coordination entre les corps de métiers. Il y avait toujours des problèmes. » Pour éviter de subir la cotraitance, plusieurs entreprises artisanales du canton de Bayon (54) ont décidé de se regrouper à la fin des années 1960, de manière informelle, sans créer de structure particulière.

«L'idée, c'était de faire un groupement libre, verbal. » Après une première expérience réussie en 1967, ils décident de «travailler en collaboration avec un maître d'œuvre. On n'a pas trouvé, on a même eu un refus. Puis on a fait la maison avec René Eury [1'architecte] sous sa coordination. Ça s'est très bien passé, on s'est trouvé des affinités et on lui a raconté, on a discuté de notre expérience réussie. Puisque l'architecte, lui, il travaille sur des plans types, il avait intérêt à travailler avec nous, surtout que les clients, à l'époque et jusqu'en 1975, passaient surtout par le maçon. » Il apparaît clairement que le processus de formulation de la stratégie provient des «impulsions déterminées [...] données dans une direction précise [...] et des réactions [qui] ont lieu face à un événement » (Thiétart, 1991). Les acteurs passent d'une stratégie désirée, mais non réalisée, à une stratégie délibérée qui se réalise dans le cadre d'un groupement formalisé. Cette stratégie de cotraitance délibérée rejoint tout à fait le principe des pseudo-concentrations de Garrette et Dussauge (1995) ou la coordination des spécialisations pour combiner des complémentarités, dans une coopération de différence au sens de Joffre et Koenig (1985).

Cette orientation stratégique a conduit ces entreprises artisanales à stabiliser les différentes relations qu'elles entretiennent avec les autres membres, d'une manière plus formelle, en nouant des accords à travers le groupement. «Le but était de créer une structure, d'être connu et de fonctionner. » Cela leur a permis de réaliser des économies d'éventail et d'exploiter des synergies, selon le principe de la stratégie de diversification, mais sans modifier leur propre organisation. « À des gens dispersés, mal informés, faiblement équipés, il [le groupement] procure un effet de taille et permet des tâches communes. Il protège contre la concurrence et les fournisseurs, facilite les opérations "synergétiques", augmente la formation par le biais des échanges. » (Louart, 1980)

La coopération entre firmes isolées permet non seulement de réduire l'isolation et les situations d'incertitude face à l'environnement, mais aussi d'acquérir une taille critique pour affronter le marché. La situation initiale demandait un rapprochement

Revue internationale P.M.E., vol. 14, n 2, 2001

(C) 2001 - Presses de l'Université du Québec

Édifice Le Delta I, 2875, boul. Laurier, bureau 450, Sainte-Foy, Québec G1V 2M2 • Tél. : (418) 657-4399 - www.puq.uquebec.ca

Tiré de : Revue internationale P.M.E., vol. 14, $\mathrm{n}^{\circ} 2$, sous la direction de Pierre-André Julien. 
des firmes dans le cadre d'un regroupement pour répondre dans un premier temps aux agressions du marché. Nous retrouvons la situation d'alliance de survie au sens de Puthod (1998), c'est-à-dire une coopération contrainte par l'environnement (survie par partage des coûts et taille critique au niveau régional): c'est une stratégie de cotraitance délibérée mais davantage dans une optique défensive qu'offensive.

SCHÉMA 2

Représentation de la modification du contexte concurrentiel par le regroupement des entreprises au sein d'un G.I.E.

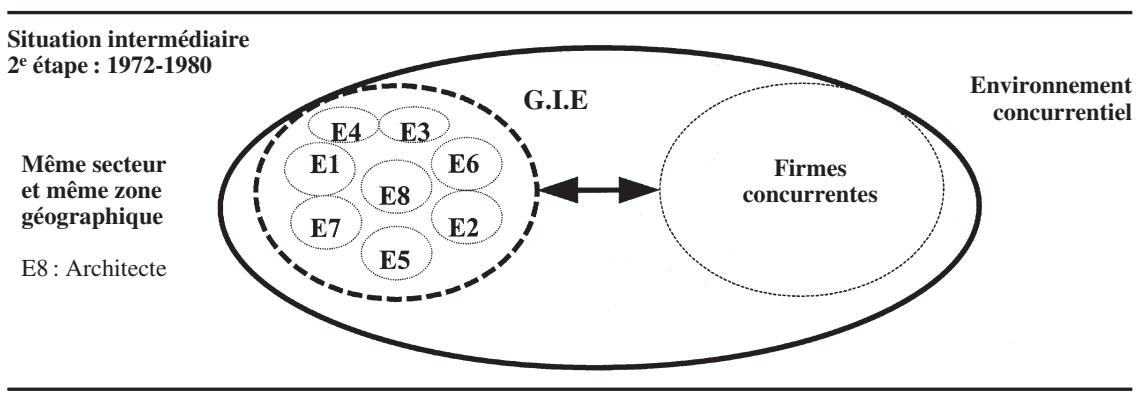

Cette position défensive est finalement confirmée par la disparition du G.I.E. : bien que la stratégie de regroupement ait été voulue par les membres, il apparaît très nettement que les relations entre les individus ont conduit à des divergences d'intérêt, voire à des conflits difficilement gérables pour le maître d'œuvre. «Dès qu'on dépasse un certain nombre de responsables, à mon avis, ce n'est plus possible de fonctionner. Il s'est créé des divergences, des problèmes entre les gens, entre certains et puis, peut-être que le maître d'ouvre n'était pas à la hauteur pour gérer les hommes: il connaissait bien son art de construire mais peut-être moins la gestion des hommes. » Le maître d'œuvre s'est trouvé débordé par la croissance du G.I.E. qu'il n'a pas su maîtriser. "C'était devenu tellement important qu'on était obligé de coopter les autres entreprises, parce qu'il y avait tellement de commandes qu'on ne pouvait pas suivre [...] et puis on a été jusqu'à une trentaine d'entreprises dans le G.I.E. [...] »

La dissolution du G.I.E. a aussi été provoquée par une modification radicale dans le marché de la construction de maisons clés en main, due à la fois à la concurrence des industries et à l'introduction de nouvelles technologies dans les matériaux et dans les techniques. 


\subsubsection{De la cotraitance délibérée à la conduite de solidarité}

Cependant, cette première expérience a permis au noyau de départ de se conforter dans l'idée que le fait d'être groupé constituait une alternative à la concurrence. Ils ont donc continué à travailler ensemble lorsque l'opportunité se présentait, ayant conservé d'excellentes relations basées sur la reconnaissance mutuelle de leur professionnalisme. «Le plus touché par la perte du marché est le maçon qui se retrouve sans travail. La finalité du nouveau groupement apparaît dans l'idée de trouver du travail à toutes les entreprises du précédent groupement et dans un souci permanent de conserver le personnel des sept entreprises : 72 salariés en tout, répartis dans cette localisation, en milieu rural. » relations :

Cette seconde étape fait apparaître deux types de stratégie dans leurs

- avec l'environnement concurrentiel, les entreprises sont perçues et fonctionnent comme un groupement orienté stratégiquement vers la cotraitance ;

- entre membres de la coopérative, les entreprises perçoivent essentiellement la nécessité de «conduites de solidarité» plus informelles, «d'ordre écosystémique, reflétant la compréhension de l'avenir en partie qui est le leur au sein d'ensembles plus larges » (Koenig, 1990 ; Bréchet, 1996). L'approche relationnelle prétend à cet égard qu'il existe une solidarité entre entreprises qui transcende leurs rapports économiques et joue un rôle essentiel dans la stratégie.

D'après Joffre et Koenig (1992), les conduites solidaires engagent les firmes concernées et d'autres acteurs de manière informelle «parce qu'avant toute chose, je considérais que, entre autres, c'était important d'apporter du travail aux uns et aux autres », dans le but de construire une évolution favorable de l'univers stratégique sur un principe de solidarité «c'est plus intéressant pour nous d'avoir des gens qui se connaissent, qui travaillent ensemble, que de travailler avec des gens qu'on connaît pas » avec un effet indirect sur les entreprises dont l'autonomie est peu affectée «tout le monde reste autonome et tout le monde reste maître de son entreprise, c'est clair». Puis, lorsqu'ils ont décidé de s'attaquer au marché de la rénovation, ils se sont spontanément concertés pour créer une nouvelle structure, avec l'intention ferme de ne pas dépasser un certain seuil : «Refaisons quelque chose sous forme de coopérative et mettons des billes dans l'opération [...] et surtout, surtout, restons à cinq ou six parce qu'une fois qu'on est trente, c'est plus gérable... on prendra des gens, on cooptera des gens, on leur sous-traitera des trucs mais on veut rester maîtres de la situation. »

Revue internationale P.M.E., vol. 14, nº 2, 2001

(C) 2001 - Presses de l'Université du Québec

Édifice Le Delta I, 2875, boul. Laurier, bureau 450, Sainte-Foy, Québec G1V 2M2 • Tél. : (418) 657-4399 - www.puq.uquebec.ca

Tiré de : Revue internationale P.M.E., vol. 14, $\mathrm{n}^{\circ} 2$, sous la direction de Pierre-André Julien. 
Cette troisième phase conduit le noyau de départ à une remise en cause de la structure du GHIS. L'échec du premier groupement formalisé leur permet de se conforter dans la nécessité d'être groupé, mais en imposant des conditions beaucoup plus strictes, à la fois dans l'organisation et dans la sélection des membres. Ils vont alors exploiter leur connaissance du groupement afin d'en conserver les points positifs et de modifier tout ce qui leur est apparu comme des erreurs stratégiques. C'est en ce sens qu'ils décident d'accentuer leurs compétences de communication entre dirigeants et de coordination des chantiers, perçu comme un avantage concurrentiel primordial. «L'idée, c'est de solutionner les problèmes avant qu'ils n'apparaissent, puisque les problèmes sont pratiquement toujours les mêmes. » La seconde nécessité qui les anime repose sur la dimension fondamentale du nombre de personnes, limité à sept, chiffre idéal selon la dynamique de groupe.

Le schéma suivant (schéma 3) permet de comprendre l'évolution des diverses structures ayant mené à la création de la coopérative. Il apparaît que la réussite de cette ultime coopération est basée sur une entreprise centrale, la SARL Jolain ; cette prépondérance de l'entreprise est confirmée par la centralisation du siège de la coopérative dans leurs locaux, par la personnalité de la gérante et par la reconnaissance des autres membres.

Nous verrons, dans la seconde partie de notre recherche, que, dans un dernier temps, la coopérative a dépassé la simple stratégie défensive pour passer à une phase de proaction, basée sur un développement des compétences de chacun et, pas seulement dans un objectif d'accroissement de performance de la coopérative, mais de chaque entreprise individuellement, voire de chaque acteur de chaque entreprise. Les entrepreneurs les plus opportunistes qui s'engagent dans la coopération considèrent que les alliances peuvent constituer un levier de développement pour la PME et ne se réduisent pas seulement à un outil de gestion des incertitudes (Puthod, 1998).

\section{Les compétences développées par les coopérants afin de gérer et contrôler leurs entreprises relationnelles}

Cette seconde section fait état des données d'enquêtes collectées auprès des membres de la coopérative "Artisans Services Bâtiment », localisée dans le canton de Vézelise (Meurthe et Moselle). Après avoir justifié le choix de ce cas, nous présentons la méthodologie de l'étude dont l'intérêt réside dans la mise en évidence d'une spécificité des entreprises artisanales dans la gestion relationnelle. La présentation des résultats de l'étude s'inscrit dans cette orientation méthodologique par une illustration fondée sur des extraits d'entretien. 
SCHÉMA 3

Analyse synchronique du développement des formes de coopération

Dates

Disparition du G.I.E., à la suite à des divergences d'opinions entre les membres et du refus de coopération de certains. Le noyau dur a continué à travailler ensemble: le projet d'association a mûri entre fin 1978 et début 1981. La coopérative, créée en 1981, devient société coopérative sous forme de SARL à personnel et capital variables en 1983.

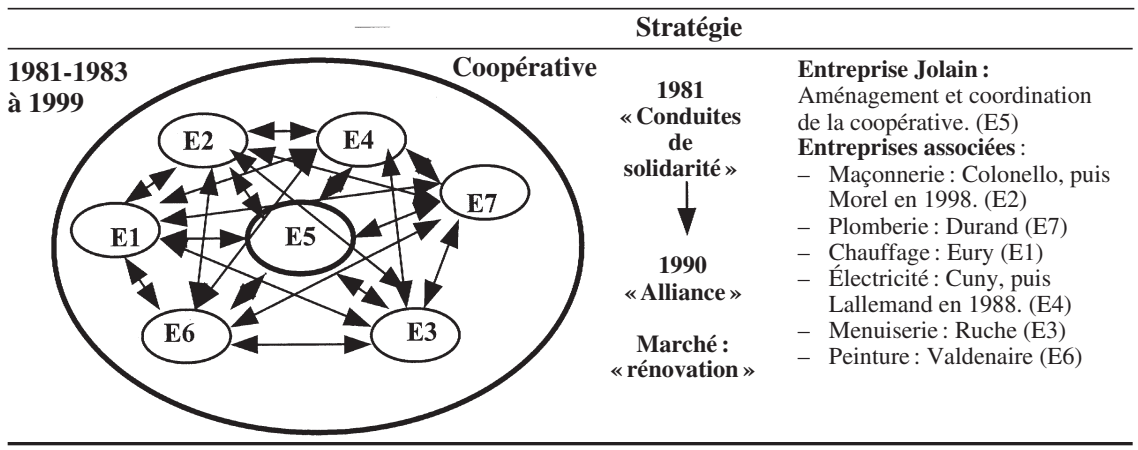

\subsection{La justification du choix de la coopérative}

Nous proposons de vérifier les critères assurant le succès d'un partenariat stratégique, déterminés par Perlmutter et Heenan (1986) et par le Standford Research Institute, en les appliquant à une coopération artisanale réussie. Ces variables ont été synthétisées dans le Stratégor (1991) et sont présentées dans le tableau 1 suivant.

La coopérative ASB correspond précisément aux critères sélectionnés précédemment, à savoir :

- la réussite : un fonctionnement formalisé depuis plus de deux décennies, presque trois si nous comptons les premières expériences du noyau de départ;

- une stratégie ex-ante : les objectifs des associés sont compatibles, chacun estimant qu'il est important d'être groupé ; 
TABLEAU 1

Conditions nécessaires à la réussite d'un partenariat stratégique

\begin{tabular}{ll}
\hline & \multicolumn{1}{c}{ Critères } \\
\hline $\begin{array}{l}\text { Perlmutter et Heenan } \\
(\mathbf{1 9 8 6 )}\end{array}$ & - Une stratégie ex-ante : une réflexion stratégique doit précéder la constitution \\
& d'une alliance afin d'évaluer la compatibilité des objectifs. \\
& - Une bonne qualité de communication et de coordination. \\
Standford Research & - Une culture commune : les partenaires doivent partager un ensemble de valeurs, \\
Institute & un style et une culture. \\
& - Des systèmes de management autonomes : l'existence d'un centre de décisions, \\
& $\begin{array}{l}\text { ayant autorité pour engager tous les partenaires, constitue l'un des facteurs de } \\
\text { succès d'une alliance. } \\
-\end{array}$ \\
& La prise en compte de certains facteurs critiques (la surspécialisation \\
& ou la perte de marge de manœuvre). \\
\hline
\end{tabular}

- une culture commune : ils sont tous artisans et nous verrons, grâce aux entretiens, qu'ils pensent partager les mêmes valeurs ;

- des systèmes de management autonomes : chaque entreprise constituant le groupement est restée autonome, le pouvoir est le même pour tous les membres-associés (un homme, une voix engendre la parité), avec un centre de décision, la coopérative ;

- la prise en compte de facteurs critiques : nous avons décidé de ne pas développer précisément ce point, mais de voir s'il existe dans la réalité de cette coopérative et comment elle la décrit.

\subsection{La méthodologie utilisée}

Par rapport à l'étude des relations entre, d'une part, des dirigeants et, d'autre part, des entreprises, il semblait important de mettre en exergue à la fois les conditions dans lesquelles s'établissent ces relations, les événements qui les ont créées et qui permettent leur poursuite mais aussi et surtout, les acteurs causals, par la prépondérance de leurs implications. L'étude de cas nous permet d'aborder à la fois la chronologie, que nous avons présentée dans la première partie, tout en évaluant les causalités locales, et de formuler une explication, en la validant auprès des individus.

Dans notre cas, la petite taille relative des entreprises, leur secteur d'activité et leur localisation géographique particulière nous permettent d'analyser le contexte, l'environnement local spécifique, l'histoire et les compétences. De plus, les acteurs étudiés sont spécifiques, non substituables et, surtout, leur participation au processus de recherche permet de négocier la place du chercheur dans l'organisation. La méthode des cas s'applique autant à une démarche exploratoire qu'à une volonté de vérification d'hypothèses (Yin et Heald, 1975 ; Avenier, 1989). L'approche nécessite généralement une présence sur le site pour l'observation du contexte, des acteurs et une collecte de données auprès de différentes sources pour permettre la triangulation.

Revue internationale P.M.E., vol. 14, $\mathrm{n}^{\circ} 2,2001$ 
La recherche sur les alliances et les coopérations est exemplaire à cet égard. Malgré une littérature pléthorique, les travaux expliquent difficilement l'articulation entre les stratégies génériques et le développement externe par alliance. Ils ne prennent pas en compte les dimensions organisationnelles, ni les conséquences humaines d'un mode de croissance contractuel particulier. (Wacheux, 1996)

Nous avons donc opté pour une démarche qualitative, qui avait pour objet d'approfondir la notion de relation dans le contexte particulier de la coopération formelle. Nous avons eu connaissance de l'existence de ce groupement par une revue bimensuelle Hommes et Métiers, produite par les chambres de métiers de Lorraine, à l'exception de la Meuse. Le contact avec les dirigeants s'est imposé de façon incrémentale par la constitution de parrains nous aménageant, au fur et à mesure, les introductions nécessaires. La rencontre avec la gérante bénévole et acteur clé de la coopérative, mademoiselle Jolain, a été facilitée par l'intervention préalable du secrétaire général de la chambre de métiers de Meurthe et Moselle, monsieur Latarche. Nous avons alors eu recours à des entretiens semi-directifs (durée moyenne : deux heures trente) que nous avons menés durant les mois de mars et avril 1999 auprès de cette personne. Ces entretiens nous ont permis d'avoir des précisions sur la structure et le fonctionnement du groupement et d'élaborer un guide d'entretien définitif et fiable grâce à une double administration du guide (test et retest). Puis, nous avons rencontré l'ensemble des membres, précédemment prévenus, lors d'une réunion mensuelle du groupe (durée : deux heures). Enfin, nous nous sommes entretenus individuellement avec chaque dirigeant, à nouveau par entretiens semi-directifs (durée: une heure trente en moyenne).

La démarche adoptée dans le guide d'entretien, basée sur les critères du tableau 1, consistait à déterminer, d'une part, les raisons de la création d'une structure formelle, à la fois en termes stratégiques, du point de vue des résultantes sur la performance de l'entreprise et au niveau relationnel, et d'autre part, les effets de cette formalisation sur les relations elles-mêmes. La collecte des données a donc procédé d'une triangulation (Jick, 1979; Stake, 1995) entre données primaires, entretiens et observation participante et des données secondaires (articles de presse). Les dialogues n'ont pas tous été retranscrits intégralement, car les premiers entretiens avec chaque personne n'ont pas été enregistrés, et ce, dans l'optique d'instaurer un climat de confiance ${ }^{3}$.

Par la suite, les enregistrements ont été traités par analyse de contenu (Weber, 1990 ; Huberman et Miles, 1991) par l'isolation d'unités thématiques, la construction

3. Certaines des personnes interrogées ont préalablement refusé l'enregistrement, à la suite d'une précédente expérience malheureuse. Il semble cependant que nous ayons rapidement réussi à surmonter leurs réticences en leur affirmant de ne rien publier qu'ils n'auraient accepté au préalable, selon le principe d'acceptation interne par les acteurs (Glaser et Strauss, 1967 ; Wacheux, 1996).

Revue internationale P.M.E., vol. 14, n 2, 2001

(C) 2001 - Presses de l'Université du Québec

Édifice Le Delta I, 2875, boul. Laurier, bureau 450, Sainte-Foy, Québec G1V 2M2 • Tél. : (418) 657-4399 - www.puq.uquebec.ca

Tiré de : Revue internationale P.M.E., vol. 14, $\mathrm{n}^{\circ} 2$, sous la direction de Pierre-André Julien. 
d'une grille de synthèse et une analyse thématique (verticale et horizontale) permettant de bâtir le rapport de synthèse. Signalons que nous n'avons pas voulu établir de hiérarchie entre les différents thèmes, puisque cette quantification, même si elle peut présenter un certain intérêt dans le cadre de notre recherche, n'autorise pas de généralisation.

\subsection{Les résultats}

En premier lieu, nous avons relevé que la coopération n'est pas envisagée ici comme un type de croissance. La finalité de la coopérative est plutôt d'affronter la conjoncture, d'éviter les risques et les incertitudes liés au marché. En second lieu, il apparaît très nettement que les acteurs tirent tous un bénéfice de la coopération; nous pouvons alors rejeter le principe du jeu à somme nulle, puisque chacun sort gagnant de l'association. Les critères présentés dans le tableau 1 permettent, selon leurs auteurs, d'établir un partenariat stratégique dans les meilleures conditions, et ce, afin d'assurer son succès. Notre approche est inverse puisqu'il s'agit, à partir d'une coopération réussie, de vérifier, en premier lieu, la présence des critères préconisés et, en second lieu, de voir s'il existe d'autres conditions essentielles à la réussite de ce partenariat et comment gérer le relationnel.

\subsubsection{Vérification des critères de réussite d'une coopération stratégique}

Nous avons constaté que tous les acteurs insistaient sur la nécessité d'être groupés pour assurer la survie de leurs entreprises, sachant que le secteur du bâtiment est soumis à des cycles conjoncturels dont les conséquences sont parfois dramatiques. C'est pourquoi il y a vraiment un objectif commun, basé sur des discussions préalables à chaque regroupement et sur une véritable réflexion stratégique quant aux solutions à mettre en place. «L'idée principale était d'apporter une solution à ce problème en proposant des travaux coordonnés, avec rapidité et de manière professionnelle. » Alors que cette stratégie peut se justifier a posteriori pour le premier groupement, elle est la condition sine qua non de la création de la coopérative. De plus, elle conduit les acteurs à formaliser leurs relations, dans le sens où ils s'imposent certaines contraintes: "la souplesse de l'entreprise d'agencement a permis de gérer les contraintes de la coopérative».

Elles sont principalement de trois ordres:

- une contrainte financière induisant une contractualisation : le statut de la coopérative passe en 1983 en SARL à personnel et à capital variables. Chacun est juridiquement et fiscalement responsable de la situation financière de la société et donc totalement impliqué dans son résultat. «Dans le G.I.E, il n'y avait pas de participation financière [...] on a tous été un 
peu pareil en se disant "on voit pas pourquoi on passerait du temps pour les autres qui attendent la bouche ouverte [...]" ». De ce fait, chaque entreprise amène des clients aux autres, car dès que l'une d'elles effectue une prestation sur un chantier quelconque, elle reste à l'affût des demandes de la clientèle sur un autre corps d'État;

- une contrainte de communication : les membres s'imposent une formalisation de la communication (le simple coup de fil informel est accompagné de télécopies et de courriers). «On travaille beaucoup, beaucoup par fax. [...] je peux téléphoner directement au peintre, mais en principe, je passe toujours par la gérante [...] je pense que c'est mieux [...] parce que si tout le monde commence à intervenir [...] non, il vaut mieux quelqu'un qui centralise les choses qui soit au courant de tout, je pense que c'est mieux $[\ldots] »$ Les réunions sont régulières, toutes les six semaines maximum, et ce, depuis 18 ans, dans un triple but de coordination des chantiers, de maintien du relationnel et de l'accroissement des connaissances. «Je suis toujours étonnée de constater qu'en 18 ans, il n'y a pratiquement jamais eu d'absent [...] Il y a une régularité systématique. C'est un besoin, un apport. »

- une contrainte de coordination : en conséquence de son engagement légal, chaque membre se doit de faire passer les chantiers de la coopérative avant les siens (priorité absolue au groupe). «On a amélioré la coordination avant la fin des travaux. [...] La coordination se fait toute seule entre nous [...] celui qui est à l'issue de la demande accompagne les autres. Une personne est désignée pour la coordination, c'est la compétence d'une personne (en général, mon frère), la reconnaissance directe des aptitudes. »

Le contrôle de la coordination et de la communication est effectué a priori, c'est-à-dire que chacun part du principe qu'il vaut mieux éviter les problèmes avant qu'ils ne se posent. À cet effet, le contact est quotidien, voire permanent. En revanche, personne ne dirige les salariés des autres entreprises et, lorsqu'un dysfonctionnement apparaît, il se règle entre dirigeants d'abord, qui répercute sur ses employés.

Cette attitude est confirmée dans la gestion de chaque entreprise. En ce qui concerne le système de management, chacun conserve son autonomie, puisque, d'une part, le statut de la coopérative impose la parité et, d'autre part, le pourcentage de chiffre d' affaires réalisé grâce au groupement ne dépasse pas les $30 \%$. Les entreprises ne sont donc pas dépendantes, ni les unes des autres, ni par rapport à la coopérative. «La gestion en interne de chaque entreprise est différente, c'est gênant, mais cela permet une liberté d'entreprise et des qualités individuelles et cela détermine une identité commune. »

Revue internationale P.M.E., vol. 14, n 2, 2001

(C) 2001 - Presses de l'Université du Québec

Édifice Le Delta I, 2875, boul. Laurier, bureau 450, Sainte-Foy, Québec G1V 2M2 • Tél. : (418) 657-4399 - www.puq.uquebec.ca

Tiré de : Revue internationale P.M.E., vol. 14, n², sous la direction de Pierre-André Julien. 
Ainsi, la localisation du siège social de la coopérative dans les locaux de l'entreprise d'agencement, même si elle souligne la place centrale de cette société, n'implique pas que celle-ci possède une certaine forme d'autorité ou de contrôle, mais plus simplement une disponibilité et une surface plus importantes.

Le centre de décision a toute autorité pour engager les partenaires et chaque entreprise envoie ses devis directement à la coopérative qui centralise toutes les informations avant de faire une proposition commune au client. "La coopérative regroupe les documents et les met en forme. Ainsi, lorsqu'un artisan envoie un devis, on prépare une première page avec l'en-tête de la coopérative.».

Enfin, la culture est réellement commune, dans le sens où, au départ, tous font partie du même secteur, le bâtiment, avec des structures relativement identiques et une conception semblable de leur rôle. Leurs objectifs sont communs aussi au regard de la formation, puisqu'ils ont tous fait des stages ensemble, afin de consolider leur communauté de fonctionnement. «L'idée du groupement, c'est que pour fonctionner pareil, il faut apprendre pareil [...] » Cela les a même poussés à informatiser leurs entreprises en même temps et avec les mêmes logiciels. Enfin, la durée de la relation a conduit à une culture commune qui apparaît dans le discours, puisque nous retrouvons beaucoup d'expression dans chaque entretien. «Ça fait un certain nombre d'années qu'on est ensemble, ça fait 25 ans, donc à force oui! [...] par rapport à des collègues, nous on a un style commun [...] y en a encore qui veulent pas entendre parler de groupement, de vouloir rester seuls et tout ça... [...] vous avez le même état d'esprit... vous avez tous à peu près la même vision, depuis le départ, puisque vous êtes ensemble, vous avez confiance les uns aux autres. »

Nous pouvons en conclure que les critères prédéterminés par Perlmutter et Heenan et par le Standford Research Institute sont effectivement nécessaires à la réussite du succès d'un partenariat. Cependant, il apparaît que si ces critères sont primordiaux, ils n'expliquent pas comment les acteurs arrivent à gérer les relations et quelles sont les compétences de contrôle et de développement du relationnel. C'est pourquoi nous avons cherché à proposer d'autres variables, tirées à la fois des entretiens réalisés et de la littérature.

\subsubsection{Mise en évidence d'autres variables nécessaires à la continuité des relations}

Il apparaît très nettement dans notre recherche que le contrôle de l'entreprise relationnelle, en l'occurrence la coopérative artisanale, est basé sur la confiance, elle-même issue de la dimension affective. Nous pouvons alors nous interroger sur la pertinence d'une gestion de l'affectif, c'est-à-dire de la modélisation des rapports émotifs. 
L'analyse de la littérature nous a permis de dégager quatre facteurs propices au développement de la confiance, que nous avons d'ailleurs pu observer dans la réalité, à savoir l'existence d'une relation antérieure réussie, la longue durée de la relation, une véritable amitié et la prépondérance du concept de réciprocité.

1. L'existence d'une expérience antérieure réussie. Selon Fenneteau et Guibert (1997), l'existence d'une relation préalable est nécessaire et déterminante afin de percevoir avec justesse la loyauté du preneur d'ordres. Dans notre cas, chaque entreprise est à la fois donneur et preneur d'ordres, puisque la réalisation de la prestation est commune à l'ensemble des associés, tandis que la personne qui obtient le contrat fait aussi partie de la structure formelle. La loyauté peut alors être vérifiée de manière continue, puisque chacun tient un double rôle. Ne pas être loyal avec les autres reviendrait à être déloyal envers soi-même.

En outre, ces auteurs estiment que lorsque la relation est précédemment inexistante, c'est la réputation qui va être déterminante, à condition, selon Orléan (1995), que les acteurs partagent les mêmes valeurs. Lors de la naissance du premier groupement, c'est effectivement cette notoriété des entreprises qui les a conduites à apprécier leur travail commun par la reconnaissance du professionnalisme des autres. «La réunion de ces personnes est basée sur l'appréciation sur le plan professionnel, ce qui est important dans la réussite d'une coopérative.»

Nous pensons que l'existence d'une expérience antérieure réussie est l'un des facteurs essentiels à la réussite de cette coopérative.

2. La durée longue de la relation. Pour Baudry (1992), la durée dans la relation conduit au développement de la confiance, «construite de façon incrémentale » et s'accumulant (Fenneteau et Guibert, 1997). Les relations entre les personnes constituant le noyau informel du départ remontent à 1967 pour les pionniers, et à 1972 pour la majorité, ce qui fait une moyenne de trente ans de connaissance. Nous estimons que c'est suffisant pour qualifier la relation de «longue durée».

De plus, cette durée permet la création d'un «savoir-faire relationnel spécifique » au sens de Asanuma (1989). «Chacun connaît les habitudes des autres [...] Si ABS et avant, le groupement, fonctionne depuis aussi longtemps, c'est parce que justement, on a privilégié les relations entre professionnels, entre entreprises, entre les associés [...]»

Dans le fonctionnement de la coopérative, cela conduit aussi à la constitution d'un savoir-faire commun, d'un apprentissage inter- et intraorganisationnel, qu'il soit incrémental ou radical: «Chaque réunion amène une question qui intéresse l'ensemble du groupe. C'est ce type de question qui conduit à faire un stage annuel en commun dans tous les domaines. »

Revue internationale P.M.E., vol. 14, nº 2, 2001

(C) 2001 - Presses de l'Université du Québec

Édifice Le Delta I, 2875, boul. Laurier, bureau 450, Sainte-Foy, Québec G1V 2M2 - Tél. : (418) 657-4399 - www.puq.uquebec.ca

Tiré de : Revue internationale P.M.E., vol. 14, $n^{\circ} 2$, sous la direction de Pierre-André Julien. 
La coopérative satisfait donc aux facteurs sélectionnés, permettant de fonder la confiance comme mécanisme de coordination entre les associés. Nous rejoignons la conception de Baudry (1994) qui estime que l'autorité, comme seul coordinateur, est insuffisante. Cette approche est évidente dans le fonctionnement de la coopérative puisque la gérante, bien que refusant d'être «directive », est unanimement reconnue comme «persuasive». «Elle reste toujours très calme, elle parle tout doucement, elle a beaucoup de persuasion, et beaucoup de ténacité [...] Elle est capable de téléphoner six fois par jour [...] pour obtenir quelque chose [...] sans jamais sortir de ses gonds.»

Mais cette notion de confiance est transcendée par une «énergie de solidarisation » (Louart, 1980), voire une véritable amitié et par la prépondérance du concept de réciprocité.

3. De la confiance à l'amitié. La confiance est un mode de coordination qui s'inscrit dans la durée, elle apparaît comme une condition nécessaire à l'établissement de relations de partenariat (Bouvier-Patron, 1995). Les entreprises s'adaptent aux évolutions qui affectent la relation en faisant en sorte qu' aucun des partenaires ne soit lésé. «Plusieurs fois, je lui ai dit, "Claude, je suis gênée, je ne comprends pas pourquoi on n'arrive pas à se transmettre des chantiers avec vous." Alors que lui trouvait son compte justement par rapport à la relation humaine. Il se ressourçait avec nous et puis ces préoccupations étaient les mêmes. »

C'est par l'échange d'informations et la réflexion en commun, formalisée par les réunions, que les dirigeants parviennent à finaliser une action collective pour faire face aux menaces pesant sur leurs entreprises. «Pour qu'un groupe s'entende bien, il faut que les personnes se connaissent, s'apprécient, fassent tous des concessions dans l'intérêt du groupe. Les réunions servent aussi à garder, à cultiver l'amitié et les relations. »

Bien que la confiance ne puisse enrayer l'apparition de conflits, c'est leur mode de résolution qui conduit au succès de ces relations. "Il y a une bonne entente, des atomes crochus mêmes, et une certaine discipline [...] Et c'est uniquement grâce à ces relations privilégiées qui sont devenues amicales [...] Pour la plupart, on se connaît; tout le monde connaît la famille, les enfants, les petits-enfants, pour certains. »

L'échange va même au-delà des rapports professionnels. «C'est comme dans un couple, il faut dialoguer, communiquer [...] C'est ce qui fait notre force, quand parfois on a des problèmes ou qu'on a sa petite déprime pour diverses raisons, quand on se retrouve ensemble dans nos réunions, on se remonte un peu le moral. [...] il y a une bonne ambiance de copains. [...] C'est pas des relations occasionnelles de travail, c'est des relations amicales. »

Revue internationale P.M.E., vol. 14, n 2, 2001

(C) 2001 - Presses de l'Université du Québec

Édifice Le Delta I, 2875, boul. Laurier, bureau 450, Sainte-Foy, Québec G1V 2M2 • Tél. : (418) 657-4399 - www.puq.uquebec.ca

Tiré de : Revue internationale P.M.E., vol. 14, $\mathrm{n}^{\circ} 2$, sous la direction de Pierre-André Julien. 
4. La gestion de la relation par le concept de réciprocité. L'observation du comportement des acteurs nous conduit à formuler une notion de relation de réciprocité, c'est-à-dire un lien existant entre des individus appartenant ou non à une même organisation, réalisant un échange équivalent et présentant un caractère mutuel. Cette réciprocité peut être formalisée ou non, mais comporte toujours une forte connotation affective :

- une recherche de chantiers interentreprises : chaque entreprise est impliquée à la fois dans ses performances et dans celles des autres. «Chacun essaie de rechercher un peu de boulot pour l'autre, si on sait qu'il a pas grand-chose à faire [...] Et puis, tout le monde joue le jeu quand même, chez nous, tout le monde joue le jeu. [...] C'est l'intérêt aussi du groupement, si tout le monde en fait autant, ça ne peut que développer une activité.»

- une régulation de l'activité par l'échange de salarié : chaque dirigeant est conscient à la fois de sa responsabilité envers ses salariés, mais aussi de la valeur du capital humain. Le maintien de l'effectif passe souvent par un échange ou un accueil de salariés d'une entreprise à l'autre. De plus, si le chantier nécessite plus de personnel, le prêt de salariés est spontané et sans risque. «Ça nous arrive souvent de nous aider mutuellement, par rapport à une activité [...] Un ouvrier part d'une entreprise pour aller dans l'autre [...] c'est fluctuant; l'an dernier au niveau de l'entreprise j'ai eu de très gros chantiers qui sont tombés et j'ai fait appel à chez Jolain. On peut très bien, tout à fait légalement se prêter des gars [...] grâce à la coopérative ASB, c'est tout à fait légal : il y a une facturation qui repasse par ASB. »

- un respect de l'identité de chacun : "Nous les sept associés, on se connaît bien, on connaît nos qualités, nos défauts, donc on agit en fonction de ça; c'est des relations humaines... donc à nous d'agir en fonction des tempéraments de chacun. [...] ne pas être directive. »

- une remise en cause réciproque : «Si les associés existent toujours, c'est parce qu'on s'est toujours remis en cause, et bien souvent collectivement [...] toujours remettre en cause les connaissances, s'adapter. »

Nous estimons, d'après les relations instaurées par ces acteurs, que la coopération est un jeu à somme non nulle, basée sur un rapport équivalent de gain et permettant de modifier les règles traditionnelles de la concurrence dans le secteur du bâtiment.

Revue internationale P.M.E., vol. 14, nº 2, 2001

(C) 2001 - Presses de l'Université du Québec

Édifice Le Delta I, 2875, boul. Laurier, bureau 450, Sainte-Foy, Québec G1V 2M2 • Tél. : (418) 657-4399 - www.puq.uquebec.ca

Tiré de : Revue internationale P.M.E., vol. 14, $\mathrm{n}^{\circ} 2$, sous la direction de Pierre-André Julien. 


\section{Conclusion}

Les limites de cette recherche proviennent, en premier lieu, du caractère qualitatif de l'étude, d'autant qu'elle porte sur une coopération « réussie » et ne peut, par conséquent, être généralisée à l'ensemble des stratégies relationnelles. En deuxième lieu, elle présente une situation particulière où les relations entre les acteurs se sont transformées au point de devenir amicales, ce qui est rarement le cas dans la pratique. En dernier lieu, elle est localisée en milieu rural pour ce qui est des sièges sociaux, tandis que les marchés sont situés dans de grandes agglomérations.

Cependant, elle permet de comprendre les facteurs de réussite et d'échec rencontrés par une coopération ancienne et alliant plus de deux entreprises. Elle propose aussi une alternative à la concurrence classique et à l'individualisme notoire des artisans. Enfin, elle démontre que la coopération n'est pas systématiquement un mode de croissance.

Il serait intéressant de reprendre cette étude dans quelques années pour assister aux différentes transmissions d'entreprises en cours et à venir, puisque plus de la moitié des associés vont atteindre l'âge de la retraite. La réussite actuelle de la coopérative étant fondée sur la particularité des relations entre ses membres, nous pouvons nous interroger sur les futurs problèmes inhérents au remplacement des dirigeants de chaque entreprise et sur la capacité des repreneurs à maintenir et à stabiliser ces relations.

\section{Bibliographie}

AldERFER, C.P. (1972), Existence, Relatedness and Growth, New York, The Free Press.

ALlAIRE, Y. et M.E. FIRSIROTU (1993), L'entreprise stratégique: penser la stratégie, Boucherville, Québec, Gaëtan Morin Éditeur.

Anastassopoulos, J.P. et al. (1985), Pour une nouvelle politique d'entreprise, Paris, Presses universitaires de France.

ARNDT, J. (1979), « Towards a concept of domesticated markets », Journal of Marketing, vol. 43, automne, p. 69-75.

AsANumA, B. (1989), «Manufacturer-supplier relationships in Japan and the concept of relation-specific skill », Journal of the Japanese and International Economies, $\mathrm{n}^{\mathrm{o}} 3$, p. 1-30.

AVENIER, M.J. (1989), « Méthodes de terrain et recherche en management stratégique», Economies et Sociétés, Série Gestion, no 14, p. 199-218.

AXELROD, R. (1984), The Evolution of Cooperation, New York, Basic.

BARREYRE, P.Y. (1968), L'impartition, politique pour une entreprise compétitive, Paris, Hachette. 
BARREYRe, P.Y. et M. Bouche (1982), «Pour une meilleure compétitivité fondée sur la solidarité interentreprises : les politiques d'impartition », Revue française de gestion, $\mathrm{n}^{\circ} 37$, septembre-octobre, p. 8-17.

BAUDRY, B. (1992), «Contrat, autorité, confiance. La relation de sous-traitance est-elle assimilable à la relation d'emploi ? », Revue Économique, vol. 43, nº 5, p. 871-894.

BAUDRY, B. (1994), L'économie des relations interentreprises, Paris, Dunod, coll. «Repères ».

BAUDRY, B. (1995), L'économie des relations interentreprises, Paris, La Découverte, coll. «Repères ».

BOUVIER-PATRON, P. (1995), «Asymétrie et symétrie relationnelle dans la relation bilatérale client-fournisseur », Économies et Sociétés, Série W, n 2, p. 18-30.

BRÉCHET, J.P. (1996), Gestion stratégique : le développement du projet d'entreprendre, Paris, Eska.

Doz, Y. et G. Hamel (1998), Alliance Advantage: The Art of Creating Value Through Partnering, Boulder, Harvard Business School Press.

DRUCKER, P. (1996), Structures et changements : balises pour un monde différent, Paris, Village mondial.

FenNeteau, H. et N. GUIBERT (1997), « Trust in buyer-seller relationship: toward a dynamic classification of the antecedents », Document de recherche, IAE, Montpellier, CREGO, 4.

GARRETTE, B. (1995), «Coopérations, alliances et compétitivité », dans M. Ingham (dir.), Management stratégique et compétitivité, Bruxelles, De Boeck Université, p. 245-260.

Garrette, B. et P. Dussauge (1995), Les stratégies d'alliances, Paris, Éditions d'Organisation.

GERVAIS, M. (1995), La stratégie de l'entreprise, Paris, Economica.

GLASER, B.G. et A.L. STRAuss (1967), The Discovery of Grounded Theory: Strategies for Qualitative Research, Chicago, Aldine.

HAMEl, D., Y. Doz et C.K. PRAHALAD (1989), «Collaborate with your competitors and win », Harvard Business Review, janvier-février, p. 133-139.

HERZBERG, H. (1966), Work and the Nature of Man, New York, The Mentor Executive.

Huberman, A. et M. MiLes (1991), Analyse des données qualitatives, Bruxelles, De Boeck Université.

JACQUEMIN, A. et B. REMICHE (1988), Coopérations entre entreprises (entreprises conjointes, stratégies industrielles et pouvoirs publics), Bruxelles, De Boeck-Université.

JENSEN, M.C. et W.H MeCKLING (1976), «Theory of the firm : managerial behavior, agency costs and ownership structure», Journal of Financial Economics, vol. 3, octobre, p. 305-360.

JICK, T. (1979), «Mixing qualitative and quantitative methods : triangulation in action », Academy of Management Journal, vol. 24, décembre, p. 602-611.

Revue internationale P.M.E., vol. 14, n 2, 2001

(C) 2001 - Presses de l'Université du Québec

Édifice Le Delta I, 2875, boul. Laurier, bureau 450, Sainte-Foy, Québec G1V 2M2 • Tél. : (418) 657-4399 - www.puq.uquebec.ca

Tiré de : Revue internationale P.M.E., vol. $14, \mathrm{n}^{\circ} 2$, sous la direction de Pierre-André Julien. 
Joffre, P. et G. KoENIG (1985), Stratégie d'entreprise - Anti-manuel, Paris, Economica. JofFre, P. et G. KoENIG (1992), Gestion stratégique, Paris, Litec.

KoEnIG, G. (1990), Management stratégique: Vision, manœuvres et tactiques, Paris, Nathan.

KoEnIG, G. (1996), Management stratégique : Paradoxes, interactions et apprentissages, Paris, Nathan.

KoEnIG, C. et G. VAN WIJK (1992), «Alliances interentreprises : le rôle de la confiance », dans A. Noël, Perspectives en management stratégique, Paris, Economica.

LOUART, P. (1980), L'artisan producteur et sous-traitant, Thèse de doctorat en sciences de gestion, Lille.

MCClelland, D. (1953), The Achievement Motive, Appleton, Century Crofts.

MARTinet, A.C. (1983), Stratégie, Paris, Vuibert, coll. « Gestion».

MAslow, A. (1954), Motivation and Personality, New York, Harper.

ORLÉAN, A. (1995), «Sur le rôle respectif de la confiance et de l'intérêt dans la constitution de l'ordre marchand », Revue du MAUSS, « À qui se fier ? Confiance, interaction et théorie des jeux », Paris, La Découverte, $n^{\circ} 4,2^{\mathrm{e}}$ semestre, p. 17-36.

Perlmutter, H. et D. Heenan (1986), «Cooperate to compete globally », Harvard Business Review, vol. 64, $\mathrm{n}^{\circ}$ 2, mars-avril, p. 136-152.

PorTER, L.W. (1964), Organizational Patterns of Managerial Job Attitudes, New York, American Foundation for Management Research.

PUTHOD, D. (1995), Les alliances de PME: stratégie de développement et implications managériales, Thèse de doctorat en sciences de gestion, Université de Savoie, GEREG.

PuTHOD, D. (1998), «L'alliance, une option stratégique permettant de contourner les dilemmes classiques de la PME», dans O. Torrès (coord.), PME, de nouvelles approches, Paris, Economica, coll. «Recherche en gestion», p. 95-104.

RING, P.S et A.H. VAN DE VEN (1992), «Structuring cooperative relationships between organizations », Strategic Management Journal, vol. 13, p. 483-498.

SiMEONI, M. (1999), La stratégie de l'artisan : une étude de sa genèse et de son élaboration par l'analyse du savoir-faire, Thèse de doctorat en sciences de gestion, Université de Corse.

STRATÉGOR (1991), Stratégie, structure, décision, identité : Politique générale d'entreprise, Paris, InterÉditions.

STAKE, R.E. (1995), The Art of Case Study Research, Thousand Oaks, CA, Sage.

TEECE, D. J. (1986), «Profiting from technological innovation : implications for integration, collaboration, licencing and public policy », Research Policy, vol. 15, $\mathrm{n}^{\circ} 6$, p. 285-305.

THIÉTART, R.A. (1991), La stratégie d'entreprise, 2e édition, Paris, McGraw-Hill.

ULRICH, D. (1983), «Governing transactions : framework for cooperative strategy », Human Resource Management, vol. 22, nos 1-2, printemps-été, p. 23-39. 
WACHEUX, F. (1993), Processus organisationnels et jeux d'acteur à l'œuvre dans les alliances entre firmes, Thèse de doctorat, Université Paris IX Dauphine.

WaCheuX, F. (1996), Méthodes qualitatives et recherche en gestion, Paris, Economica.

Weber, R. P. (1990), Basic Content Analysis, Londres, Sage University Paper, no 49.

WILlIAMSON, O.E. (1975), Markets and Hierarchies : Analysis and Antitrust Implications, New York, The Free Press.

WiLliamson, O.E. (1985), The Economic Institutions of Capitalism, New York, The Free Press.

YIN, R.K. et K.A HEALD (1975), «Using the case survey method to analyze policy studies », Administrative Science Quarterly, vol. 20, p. 371-381.

ZAHEER, A. et N. VENKATRAM (1995), «Relational governance as an interorganizational strategy : an empirical test of the role of trust in economic exchange », Strategic Management Journal, vol. 16, no 5, juin, p. 373-392.

ZEITHAML, C.P et V.A. ZEITHAML (1984), «Environmental management : revisiting the marketing perspective », Journal of Marketing, vol. 47, printemps, p. 46-53.

Revue internationale P.M.E., vol. 14, $\mathrm{n}^{\circ}$ 2, 2001 